\title{
Eisenmangel, Thrombozytose und Thromboembolie
}

\author{
Rayko Evstatiev
}

Eingegangen: 3. April 2016 / Angenommen: 5. August 2016 / Online publiziert: 28. September 2016 (c) Der/die Autor(en) 2016. Dieser Artikel ist eine Open-Access-Publikation.

Zusammenfassung Eisenmangel, die häufigste Mangelerscheinung weltweit, ist häufig mit einer reaktiven Thrombozytose assoziiert. Während eine sekundäre Thrombozytose generell als harmlos angesehen wird, mehren sich die Hinweise dafür, dass eine Thrombozytenanzahlerhöhung insbesondere bei Eisenmangel mit einem erhöhten Thromboembolierisiko sowohl im arteriellen als auch im venösen System assoziiert sein kann. Hier werden die Mechanismen der eiseninduzierten Thrombozytose geschildert und die klinischen Konsequenzen insbesondere bei Patienten mit chronisch entzündlichen Darmerkrankungen, chronischer Niereninsuffizienz oder malignen Erkrankungen analysiert. Wir hypothetisieren, dass Eisenmangel ein bis jetzt unterschätzter Thromboembolierisikofaktor darstellt, und dass Eisenersatztherapie eine effektive präventive Strategie sein kann.

Schlüsselwörter Eisenmangel · Thrombozytose · Anämie $\cdot$ Thromboembolie $\cdot$ Thrombose

\section{Iron deficiency, thrombocytosis and} thromboembolism

Summary Iron deficiency, the most common nutritional deficiency worldwide, is often associated with reactive thrombocytosis. Although secondary thrombocytosis is commonly considered to be harmless, there is accumulating evidence that elevated platelet counts, especially in the setting of iron deficiency, can lead to an increased thromboembolic risk in both arterial and venous systems. Here we present the

\section{Dr. R. Evstatiev ( $\square)$}

Division of Gastroenterology and Hepatology, Department of Internal Medicine III, Medical University of Vienna, Währinger Gürtel 18-20, 1090 Wien, Österreich rayko.evstatiev@meduniwien.ac.at mechanisms of iron deficiency-induced thrombocytosis and summarize its clinical consequences especially in patients with inflammatory bowel diseases, chronic kidney disease or cancer. We hypothesize that iron deficiency is an underestimated thromboembolic risk factor, and that iron replacement therapy can become an effective preventive strategy in a variety of clinical settings.

Keywords Iron deficiency · Thrombocytosis · Anemia • Thromboembolism $\cdot$ Thrombosis

Eisenmangel ist der welthäufigste nutritive Mangel und betrifft bis zu einem Drittel der Weltbevölkerung [1]. Eine Reihe von wichtigen Proteinen wie Sauerstoffträger, Proteine der respiratorischen Kette, Zytochrome und DNA-Synthese- und -Reparaturenzyme enthalten Eisen; daher sind die klinischen Ausprägungen des Eisenmangelsyndroms mannigfaltig und umfassen Anämie, neurologische Störungen inkl. neuropsychiatrische Entwicklungsstörungen, Depressionen und „restless legs“ Syndrom, Herzmuskelschwäche sowie trophische Haut- und Nagelstörungen [2, 3]. Eine der wichtigsten Ursachen für Eisenmangel ist der Blutverlust - daher ist es evolutionär sinnvoll, dass bei Eisenmangel adaptive Mechanismen eingeschaltet werden, wodurch ein weiterer Blutverlust verhindert wird. Ein solcher potentieller Mechanismus könnte die eisenmangelinduzierte Thrombozytose darstellen.

Die zelluläre Gerinnung ist ein essentieller Schutzmechanismus, wodurch ein Blutverlust über beschädigte Blutgefäße verhindert wird und die Voraussetzungen für eine Wundheilung geschaffen werden. Zelluläre Gerinnungssysteme existieren in praktisch allen Mehrzellern und reichen von primitiven multifunktionellen Zelltypen bei Anneliden, Echinodermaten oder Arthropoden, die neben Koagulations- 


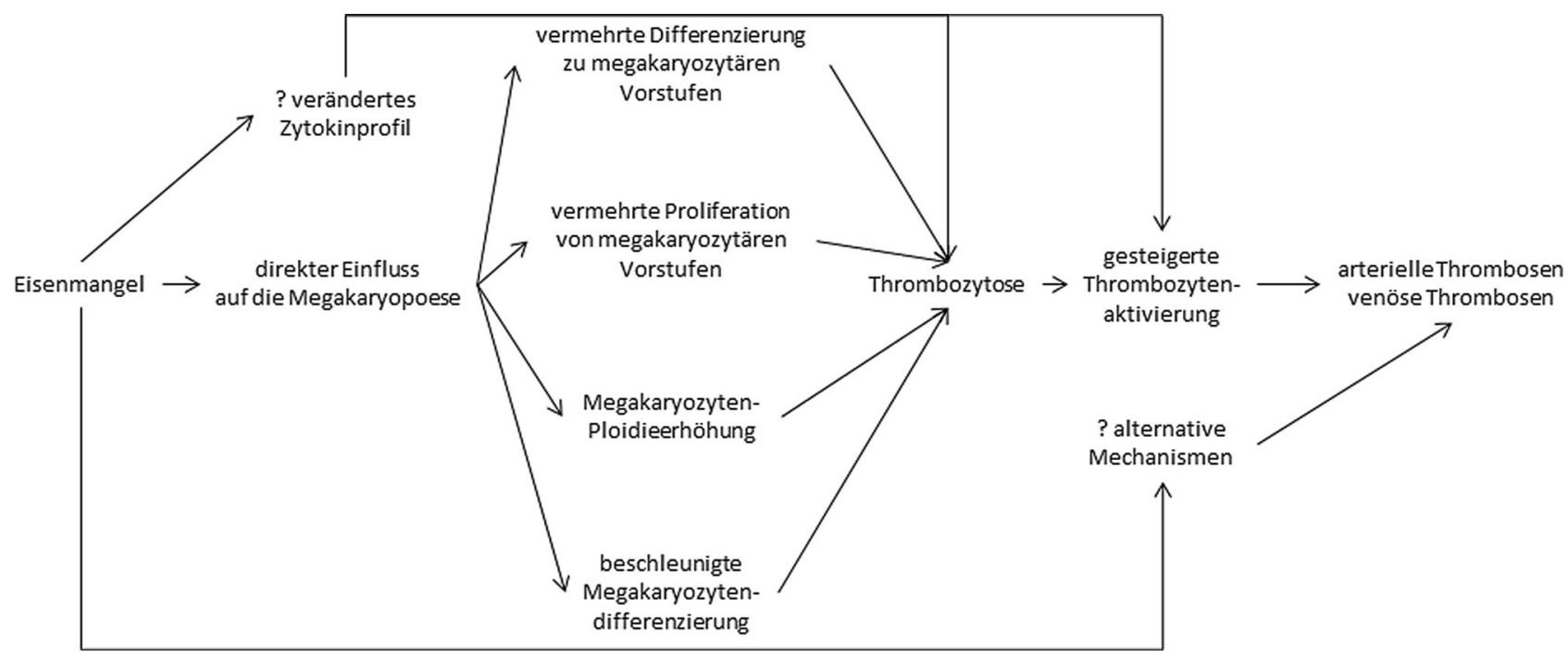

Abb. 1 Der Zusammenhang zwischen Eisenmangel, Thrombozytose und Thrombose-ein Modell

aufgaben auch der unspezifischen Abwehr dienen, über die spezialisierten kernhaltigen Thrombozyten der Fische, Amphibien, Reptilien und Vögel bis zu den hochspezialisierten kernlosen Blutplättchen bei Säugetieren $[4,5]$. Die Thrombozyten bei Säugetieren werden von den Megakaryozyten gebildet, die die seltensten aber größten Knochenmarkzellen darstellen. Die Megakaryozyten stammen von den hämatopoetischen Stammzellen ab, die über einer proliferativen Phase und anschließend einer endomitotischen Phase in reife polyploide plättchenproduzierende Zellen differenzieren. Im Rahmen der Endomitose entsteht eine Steigerung der Ploidie aufgrund einer inkompletten Mitose mit Abbruch der Zytokinese [6-8]. Durch die Endomitose wird eine Expansion des Zytoplasma ermöglicht, ohne dass die Zelle unnötige Energie für die Ausführung aller Schritte der Zellteilung verschwendet [9]; man könnte spekulieren, dass dadurch auch Eisen gespart werden könnte [10].

Eine Erhöhung der Thrombozytenanzahl kann entweder Folge der Vermehrung eines aberranten Stammzellklons sein wie bei der essentiellen Thrombozytose, Polycythaemia vera oder bei anderen myeloproliferativen Erkrankungen (primäre Thrombozytose) oder begleitend bei Verletzungen, postoperativ, bei akuten oder chronischen Infektionen, malignen Erkrankungen oder chronischen entzündlichen Erkrankungen (wie chronisch entzündliche Darmerkrankungen oder Erkrankungen des rheumatischen Formenkreises) oder nach einer Splenektomie auftreten (sekundäre oder reaktive Thrombozytose) [11-14]. Eisenmangel ist eine anerkannte Ursache für reaktive Thrombozytose auch ohne Entzündung. Zahlreiche klinische Studien [13, 15-23] bekräftigen diesen Zusammenhang. In Tiermodellen konnte der Einfluss des Eisenmangels auf die Entstehung einer Thrombozytose unabhängig von anderen Faktoren bestätigt werden [10, 24-28]. In retrospektiven Studien liegt die Häufigkeit der Thrombozytose bei Eisenman- gelanämie zwischen 13-28\% [23, 29]. Die Ausprägung der Thrombozytose ist typischerweise mild, und eine Eisensubstitutionstherapie führt zu einer Normalisierung der Thrombozytenanzahl [16, 21, 22, 28, 30, 31]. Der Zusammenhang ist allerdings komplex, und ein schwerer Eisenmangel kann ebenfalls eine Thrombopenie verursachen [129]. Diese Komplexität lässt sich auch in zwei genetischen Tiermodellen des Eisenmangels veranschaulichen, nämlich der BelgradeRatte [130], die eine Mutation des Eisenimporters DMT1 aufweist, sowie der Mask-Maus [131], die im negativen Hepcidin-Regulatorgen TMPRSS6 (Matriptase-2) mutiert ist. Beide Tiermodelle weisen eine Thrombopenie auf. Der Mechanismus der eisenmangelinduzierten Thrombozytose ist nach wie vor ungeklärt (Abb. 1). Ein indirekter Effekt auf die Thrombopoese über Zytokine und hämatopoetische Faktoren erscheint eher unwahrscheinlich. Der Thrombopoetinspiegel, der wichtigste Stimulators der Thrombopoese, korreliert nicht gut mit der Ausprägung der Thrombozytose [10, 32-34]. Weitere thrombopoetische Faktoren wie IL-6 und IL-11 sind unverändert im Rahmen der eisenmangelinduzierten Thrombozytose. Das einzige thrombopoetische Zytokin, dessen Konzentration erhöht ist, ist das Erythropoetin [10, 32], dessen Rolle in der Thrombopoese sehr umstritten ist. Erythropoetin erhöht die Plättchenanzahl in vivo [35], und stimuliert (synergistisch mit weiteren megakaryopoetischen Faktoren) die Megakaryozytenproliferation in vitro [36]. Allerdings unterstützt EPO alleine kaum das Megakaryozytenwachstum [37], da reifende Megakaryozyten den EPO Rezeptor verlieren [38]. Eine ursprünglich vermutete direkte Wirkung über den Thrombopoetinrezeptor c-mpl [39] konnte nicht nachgewiesen werden [37, 40]. Der EPO Spiegel korreliert schlecht mit der Plättchenanzahl und kann auch in der Abwesenheit einer Thrombozytose erhöht sein [32, 37, 40]. Eine Eisensubstitution bei Thrombozytose normalisiert die Thrombozytenan- 
Tab. 1 Fallberichte über arterielle thromboembolische Komplikationen bei Patienten mit Eisenmangel, Anämie und Thrombozytose

\begin{tabular}{|c|c|c|c|c|c|c|c|}
\hline $\begin{array}{l}\text { Thrombo-embolisches } \\
\text { Ereignis }\end{array}$ & Geschlecht & Alter & $\begin{array}{l}\text { Hämoglobin } \\
\text { [g/dl] }\end{array}$ & $\begin{array}{l}\text { Thrombozyten } \\
{[G / 1]}\end{array}$ & $\begin{array}{l}\text { Ätiologie des Eisen- } \\
\text { mangels }\end{array}$ & Weitere Risikofaktoren & Referenz \\
\hline Ischämischer Insult & Männlich & 42 Jahre & 4,8 & 1016 & Hämorrhoidenblutung & Keine angegeben & [95] \\
\hline Ischämischer Insult & Weiblich & 42 Jahre & $\begin{array}{l}27 \% \text { (Häma- } \\
\text { tokrit) }\end{array}$ & 1736 & Uterusleiomyom & Keine angegeben & [96] \\
\hline $\begin{array}{l}\text { Thrombose der A. ce- } \\
\text { rebri media und der A. } \\
\text { centralis retinae, Throm- } \\
\text { bose der A. dorsalis } \\
\text { pedis, Pulmonalembolie }\end{array}$ & Weiblich & 26 Jahre & 9,2 & 850 & Uterusleiomyom & Postoperativ & [97] \\
\hline Ischämischer Insult & Weiblich & 27 Monate & 4,5 & 728 & Mangelernährung & Keine angegeben & [98] \\
\hline Ischämischer Insult & Weiblich & 45 Jahre & 5,9 & 171 & Uterusleiomyom & Keine angegeben & [99] \\
\hline Ischämischer Insult & Weiblich & 14 Jahre & 4,0 & 65 & Kolonpolypen & Keine angegeben & {$[100]$} \\
\hline Ischämischer Insult & Weiblich & 30 Jahre & 6,7 & 1050 & Uterusleiomyom & Keine angegeben & [101] \\
\hline Ischämischer Insult & Weiblich & 20 Jahre & 6,3 & 544 & Hypermenorrhoe & Keine angegeben & [102] \\
\hline $\begin{array}{l}\text { Thrombose der A. caro- } \\
\text { tis communis, ischämi- } \\
\text { scher Insult }\end{array}$ & Weiblich & 39 Jahre & 7,1 & 652 & Uterusleiomyom & Keine angegeben & [102] \\
\hline $\begin{array}{l}\text { Thrombose der A. caro- } \\
\text { tis interna, ischämischer } \\
\text { Insult }\end{array}$ & Weiblich & 44 Jahre & 6,6 & 666 & Hypermenorrhoe & Keine angegeben & [102] \\
\hline $\begin{array}{l}\text { Thrombose der A. cen- } \\
\text { tralis retinae }\end{array}$ & Weiblich & 13 Jahre & 5,5 & 535 & Mangelernährung & Keine angegeben & [103] \\
\hline Ischämischer Insult & Weiblich & 11 Monate & 12,6 & 693 & Nicht angegeben & Keine angegeben & [104] \\
\hline Ischämischer Insult & Weiblich & 18 Monate & 11,4 & 373 & Nicht angegeben & Keine angegeben & [104] \\
\hline Ischämischer Insult & Weiblich & 18 Monate & 9,8 & 531 & Mangelernährung & Keine angegeben & [104] \\
\hline Ischämischer Insult & Weiblich & 23 Monate & 2,7 & 217 & Mangelernährung & Keine angegeben & [105] \\
\hline $\begin{array}{l}\text { Thrombose der A. cen- } \\
\text { tralis retinae }\end{array}$ & Weiblich & 50 Jahre & 7,3 & 390 & Hypermenorrhoe & Keine angegeben & [106] \\
\hline $\begin{array}{l}\text { Embolie der A. ulnaris, } \\
\text { weiters Pulmonalembo- } \\
\text { lie }\end{array}$ & Weiblich & 32 Jahre & 8,7 & 865 & Hypermenorrhoe & Keine angegeben & [18] \\
\hline $\begin{array}{l}\text { Thrombose der A. caro- } \\
\text { tis, ischämischer Insult }\end{array}$ & Weiblich & 48 Jahre & 7,6 & 1139 & Nicht angegeben & Keine angegeben & [18] \\
\hline Ischämischer Insult & Weiblich & 17 Jahre & $\begin{array}{l}\text { Nicht ange- } \\
\text { geben }\end{array}$ & 481 & Colitis indeterminata & $\begin{array}{l}\text { Colitis indeterminata, } \\
\text { Protein S Mangel, Faktor V } \\
\text { Leiden Heterozygotie }\end{array}$ & [107] \\
\hline Ischämischer Insult & Männlich & 32 Jahre & 7,6 & 2254 & Ulcus duodeni & Postoperativ & [108] \\
\hline $\begin{array}{l}\text { Thromboembolien mul- } \\
\text { tipler Beinvenenarterien }\end{array}$ & Weiblich & 47 Jahre & $\begin{array}{l}\text { Nicht ange- } \\
\text { geben }\end{array}$ & 1237 & Morbus Crohn & Morbus Crohn & [109] \\
\hline $\begin{array}{l}\text { Thrombose der Aorta de- } \\
\text { scendens, embolischer } \\
\text { Verschluss mehrerer } \\
\text { Beinarterien }\end{array}$ & Weiblich & 42 Jahre & 7,7 & 465 & Hypermenorrhoe & Keine angegeben & [110] \\
\hline $\begin{array}{l}\text { Thrombose des Arcus } \\
\text { aortae, ischämischer } \\
\text { Insult }\end{array}$ & Weiblich & 49 Jahre & 8,4 & 567 & Hypermenorrhoe & Keine angegeben & [110] \\
\hline $\begin{array}{l}\text { Thrombose der A. caro- } \\
\text { tis interna, ischämischer } \\
\text { Insult }\end{array}$ & Weiblich & 41 Jahre & 7,5 & 450 & Hypermenorrhoe & Keine angegeben & [111] \\
\hline Ischämischer Insult & Weiblich & 42 Jahre & 5,3 & 260 & Uterusleiomyom & Keine angegeben & [112] \\
\hline Ischämischer Insult & Weiblich & 42 Jahre & 11,6 & 429 & Uterusleiomyom & Keine angegeben & [112] \\
\hline
\end{tabular}

zahl auch bei Patienten, die EPO erhalten [21, 22, 41]. Es scheint daher, dass Eisenmangel einen direkten Effekt auf die Thrombopoese ausübt. Diskrete Veränderungen der Megakaryopoese mit Expansion von Megakaryozytenvorstufen im Knochenmark, erhöhter Ploidie, beschleunigter Differenzierung und
Proplättchenbildung konnten in Tierexperimenten sowie in hämatopoetischen Stammzellen und megakaryozytären Zelllinien gezeigt werden [10, 25, 42]. Auf molekularer Ebene könnte diese beschleunigte Differenzierung durch HIF (hypoxia-inducible factor)- 


\section{themenschwerpunkt}

Tab. 2 Fallberichte über venöse thromboembolische Komplikationen bei Patienten mit Eisenmangel, Anämie und Thrombozytose

\begin{tabular}{|c|c|c|c|c|c|c|c|}
\hline $\begin{array}{l}\text { Thrombo-embolisches } \\
\text { Ereignis }\end{array}$ & Geschlecht & Alter & $\begin{array}{l}\text { Hämoglobin } \\
\text { [g/dl] }\end{array}$ & $\begin{array}{l}\text { Thrombozyten } \\
{[G / /]}\end{array}$ & $\begin{array}{l}\text { Ätiologie des Eisenman- } \\
\text { gels }\end{array}$ & Weitere Risikofaktoren & Referenz \\
\hline $\begin{array}{l}\text { Thrombose der V. cen- } \\
\text { tralis retinae }\end{array}$ & Weiblich & 44 Jahre & 6,2 & 280 & Post partum & Keine angegeben & [113] \\
\hline $\begin{array}{l}\text { Pulmonalembolie, } \\
\text { Thrombose der A. ce- } \\
\text { rebri media und der } \\
\text { A. centralis retinae, } \\
\text { Thrombose der A. dorsa- } \\
\text { lis pedis }\end{array}$ & Weiblich & 26 Jahre & 9,2 & 850 & Uterusleiomyom & Postoperativ & [97] \\
\hline $\begin{array}{l}\text { Zerebrale Sinusvenen- } \\
\text { thrombose }\end{array}$ & Männlich & 22 Monate & 4,0 & 1000 & Mangelernährung & Keine angegeben & [114] \\
\hline $\begin{array}{l}\text { Zerebrale Sinusvenen- } \\
\text { thrombose }\end{array}$ & Weiblich & 48 Jahre & 6,8 & 125 & Uterusleiomyom & Keine angegeben & [99] \\
\hline $\begin{array}{l}\text { Thrombose der V. cen- } \\
\text { tralis retinae }\end{array}$ & Weiblich & 21 Jahre & 6,5 & Nicht angegeben & Anorexia nervosa & Keine angegeben & [115] \\
\hline $\begin{array}{l}\text { Zerebrale Sinusvenen- } \\
\text { thrombose }\end{array}$ & Weiblich & 18 Monate & 6,7 & 972 & Nicht angegeben & Keine angegeben & [104] \\
\hline $\begin{array}{l}\text { Zerebrale Sinusvenen- } \\
\text { thrombose }\end{array}$ & Weiblich & 12 Monate & 7,7 & 380 & Nicht angegeben & Keine angegeben & [104] \\
\hline $\begin{array}{l}\text { Zerebrale Sinusvenen- } \\
\text { thrombose }\end{array}$ & Männlich & 6 Monate & 4,5 & 657 & Mangelernährung & Keine angegeben & [104] \\
\hline $\begin{array}{l}\text { Zerebrale Sinusvenen- } \\
\text { thrombose }\end{array}$ & Männlich & 4 Jahre & 8,3 & 1194 & Nicht angegeben & Keine angegeben & [116] \\
\hline $\begin{array}{l}\text { Thrombose der V. cen- } \\
\text { tralis retinae }\end{array}$ & Weiblich & 37 Jahre & 9,4 & Normal & Nicht angegeben & Keine angegeben & [106] \\
\hline $\begin{array}{l}\text { Zerebrale Sinusvenen- } \\
\text { thrombose }\end{array}$ & Weiblich & 27 Monate & 7,0 & 248 & Mangelernährung & Keine angegeben & [117] \\
\hline $\begin{array}{l}\text { Zerebrale Sinusvenen- } \\
\text { thrombose }\end{array}$ & Weiblich & 9 Monate & 6,6 & 586 & Nicht angegeben & Keine angegeben & [117] \\
\hline $\begin{array}{l}\text { Zerebrale Sinusvenen- } \\
\text { thrombose }\end{array}$ & Männlich & 19 Monate & 6,9 & 408 & Mangelernährung & Keine angegeben & [117] \\
\hline $\begin{array}{l}\text { Zerebrale Sinusvenen- } \\
\text { thrombose }\end{array}$ & Männlich & 14 Jahre & 7,4 & 289 & Nicht angegeben & Dehydrierung & [118] \\
\hline $\begin{array}{l}\text { Zerebrale Sinusvenen- } \\
\text { thrombose }\end{array}$ & Weiblich & 38 Jahre & 6,8 & 560 & Nicht angegeben & Keine angegeben & [119] \\
\hline $\begin{array}{l}\text { Zerebrale Sinusvenen- } \\
\text { thrombose }\end{array}$ & Weiblich & 18 Jahre & 5,7 & 640 & Nicht angegeben & Keine angegeben & [119] \\
\hline $\begin{array}{l}\text { Zerebrale Sinusvenen- } \\
\text { thrombose }\end{array}$ & Weiblich & 16 Jahre & $\begin{array}{l}\text { Nicht ange- } \\
\text { geben }\end{array}$ & 429 & Morbus Crohn & $\begin{array}{l}\text { Morbus Crohn, Prothrom- } \\
\text { binmutation }\end{array}$ & [107] \\
\hline $\begin{array}{l}\text { Zerebrale Sinusvenen- } \\
\text { thrombose }\end{array}$ & Weiblich & 12 Jahre & $\begin{array}{l}\text { Nicht ange- } \\
\text { geben }\end{array}$ & 756 & Morbus Crohn & Morbus Crohn & [107] \\
\hline $\begin{array}{l}\text { Zerebrale Sinusvenen- } \\
\text { thrombose }\end{array}$ & Weiblich & 18 Jahre & $\begin{array}{l}\text { Nicht ange- } \\
\text { geben }\end{array}$ & 837 & Morbus Crohn & Morbus Crohn & [107] \\
\hline $\begin{array}{l}\text { Zerebrale Sinusvenen- } \\
\text { thrombose }\end{array}$ & Männlich & 55 Jahre & 8,7 & 272 & Meläna & Keine angegeben & [120] \\
\hline $\begin{array}{l}\text { Zerebrale Sinusvenen- } \\
\text { thrombose }\end{array}$ & Männlich & 18 Monate & $\begin{array}{l}22 \% \text { (Häma- } \\
\text { tokrit) }\end{array}$ & 282 & Nicht angegeben & Keine angegeben & [121] \\
\hline $\begin{array}{l}\text { Zerebrale Sinusvenen- } \\
\text { thrombose, Pulmona- } \\
\text { lembolie }\end{array}$ & Weiblich & 63 Jahre & 3,4 & Nicht angegeben & Nicht angegeben & Keine angegeben & [122] \\
\hline $\begin{array}{l}\text { Zerebrale Sinusvenen- } \\
\text { thrombose }\end{array}$ & Männlich & 9 Monate & 4,1 & 866 & Mangelernährung & Keine angegeben & [123] \\
\hline $\begin{array}{l}\text { Zerebrale Sinusvenen- } \\
\text { thrombose }\end{array}$ & Weiblich & 43 Jahre & 7,6 & 260 & Uterusleiomyom & Postoperativ & [124] \\
\hline $\begin{array}{l}\text { Zerebrale Sinusvenen- } \\
\text { thrombose }\end{array}$ & Weiblich & 47 Jahre & 7,6 & Nicht angegeben & Adenomyosis uteri & Keine angegeben & [125] \\
\hline
\end{tabular}


Tab. 2 (Fortsetzung)

\begin{tabular}{llll|l|l|l|l|}
$\begin{array}{l}\text { Thrombo-embolisches } \\
\text { Ereignis }\end{array}$ & Geschlecht & Alter & $\begin{array}{l}\text { Hämoglobin } \\
{[\mathrm{g} / \mathrm{dl}]}\end{array}$ & $\begin{array}{l}\text { Thrombozyten } \\
{[\mathrm{G} / \mathrm{l}]}\end{array}$ & $\begin{array}{l}\text { Ätiologie des Eisenman- } \\
\text { gels }\end{array}$ & Weitere Risikofaktoren & Referenz \\
$\begin{array}{l}\text { Zerebrale Sinusvenen- } \\
\text { thrombose }\end{array}$ & Weiblich & 13 Jahre & 5,1 & 483 & Hypermenorrhoe & Keine angegeben & {$[126]$} \\
$\begin{array}{l}\text { Zerebrale Sinusvenen- } \\
\text { thrombose }\end{array}$ & Weiblich & 29 Jahre & 6,5 & 280 & Nicht angegeben & $\begin{array}{l}\text { Minimal erniedrigte Prote- } \\
\text { in C und Protein S-Aktivität }\end{array}$ & {$[127]$} \\
$\begin{array}{l}\text { Zerebrale Sinusvenen- } \\
\text { thrombose }\end{array}$ & Weiblich & 4 Jahre & 7,7 & 1082 & Nicht angegeben & Keine angegeben & {$[128]$} \\
\hline
\end{tabular}

$2 \alpha$ und VEGF (vascular endothelial growth factor)-A mediiert sein [42].

Weiters ist die klinische Bedeutung der reaktiven Thrombozytose unklar. Während die primäre Thrombozytose ein unumstrittener prothrombogener Risikofaktor darstellt, gilt die sekundäre Thrombozytose generell als harmlos [13]. Obwohl große prospektive Studien größtenteils fehlen, konnte in mehreren Analysen gezeigt werden, dass eine Thrombozytose in unterschiedlichen Situationen wie postoperativ [43, 44], posttraumatisch [45], bei Intensivpatienten [46, 47], SIRS (systemic inflammatory response syndrome) [48] oder Malignompatienten [49-51] sehr wohl ein unabhängiger Risikofaktor für thromboembolische Komplikationen mit berichteten OR (odds ratio) von 1,13-1,78 bzw. HR (hazard ratio) von 3,50-5,3 darstellen kann. In vitro konnte eine erhöhte Plättchenaktivierung gezeigt werden [52-54].

Auch Eisenmangel und die eisenmangelinduzierte Thrombozytose wurden mit einem erhöhten Thromboembolierisiko in Verbindung gebracht. In retrospektiven Studien scheint die Wahrscheinlichkeit, einen ischämischen Insult $\mathrm{zu}$ erleiden, bei Eisenmangelanämie höher zu sein (OR 1,49-6,3) [55-59]. Auch für venöse thromboembolische Ereignisse wurden ähnliche Ergebnisse berichtet (OR 1,43) [60]; des Weiteren erhöht ein Eisenmangel das Risiko eines Wiederauftretens einer venösen Thromboembolie [61]. Die Eisenmangelanämie ist ein vermuteter Risikofaktor für die zerebrale Sinusthrombose [62]. Es gibt zahlreiche Fallberichte über thromboembolische Komplikationen bei Eisenmangel und eisenmangelinduzierter Thrombozytose sowohl im arteriellen (Tab. 1) als auch im venösen System (Tab. 2). Nichtsdestotrotz mangelt es nach wie vor an großen Studien mit ausreichendem Evidenzgrad, um zu bestätigen, dass Eisenmangel ein Risikofaktor für thromboembolische Ereignisse darstellt. Tierstudien, in denen der isolierte Einfluss des Eisenmangels und der eisenmangelinduzierten Thrombozytose auf die Entstehung von Thrombosen unabhängig von Komorbiditäten untersucht wird, fehlen ebenfalls. Eine Assoziation zwischen Eisenmangel und Thrombosen könnte die Grundlage eines neuen Therapiekonzepts darstellen, das potentiell tödlichen thromboembolischen Komplikationen entgegenwirken kann. Die Rolle von Eisenmangel und eisenmangelinduzierter Thrombozytose könnte bei diversen Erkrankungsgruppen wie chronisch entzündlichen Darmerkrankungen, chroni- scher Niereninsuffizienz und malignen Erkrankungen in diesem Zusammenhang relevant sein.

\section{Eisenmangel, Thrombozytose und chronisch ent- zündliche Darmerkrankungen}

Sowohl Eisenmangel als auch Thrombozytose sind häufige Begleiterscheinungen bei den chronisch entzündlichen Darmerkrankungen (CED) Morbus Crohn und Colitis ulcerosa und sind Ausdruck einer aktiven Erkrankung. Weiters stellt eine aktive CED einen Risikofaktor für thromboembolische Komplikationen mit einem RR von 4,5 dar [63-65]. Eisenmangel und Eisenmangelanämie, die häufigsten extraintestinalen Komplikationen bei CED, entstehen in erster Linie durch chronische Blutung aus Darmschleimhautulzerationen, aber auch durch verminderter Eisenzufuhr und -Aufnahme [66, 67]. Die Ausprägung der CED-assoziierten Thrombozytose korreliert mit der entzündlichen Aktivität [68, 69] und ist einerseits durch die Wirkung proinflammatorischer Zytokine wie Interleukin 6 bedingt [70], andererseits scheint Eisenmangel ebenfalls eine Rolle zu spielen [21, 69, 71]. Die erhöhte Plättchenanzahl bei CED ist ferner mit einer erhöhten Aktivität verbunden [72]. In der randomisierten Studie ThromboVIT konnte gezeigt werden, dass eine intravenöse Eisentherapie eine Normalisierung der Thrombozytenanzahl und -Aktivität erzielen kann [22]. Somit könnte die Behandlung des Eisenmangels eine weitere Säule der Thromboembolieprävention darstellen, wobei prospektive Bestätigungstudien notwendig sind.

\section{Eisenmangel, Thrombozytose und Erythropoetin bei chronischer Niereninsuffizienz}

Die renale Anämie ist eine der wichtigsten Komplikationen der chronischen Niereninsuffizienz. Sie hat eine multifaktorielle Genese, wobei die verminderte Erythropoetinaktivität der führende pathogenetische Faktor ist. Eine Behandlung mit Erythropetin und Analoga ist der Eckpfeiler der Therapie der renalen Anämie. Paradoxerweise führt aber eine Normalisierung des Hämoglobins mittels Erythropoese-stimulierenden Agenten zu einer erhöhten Morbidität und Mortalität [73-76], wobei ein gesteigertes Risiko für kadiovaskuläre Ereignisse inklusive venöse Thromboembolien, Myokardinfarkte und Shuntthrombosen dafür verantwortlich gemacht wird. 
Ein weiterer wesentlicher pathogenetischer Faktor in der Entstehung der renalen Anämie ist der Eisenmangel. Eisenmangel bei chronischer Niereninsuffizienz entsteht durch Blutverlust (im Rahmen der Hämodialyse, über den Gastrointestinaltrakt und aufgrund von häufigen Blutabnahmen oder chirurgischen Eingriffen wie Shuntanlagen) und durch verminderte Eisenresorption (z.B. durch Medikamenteninteraktion wie Phosphatbinder). Aufgrund der chronischen Entzündung kommt es ferner zu einer Hepcidin-mediierten Eisenverwertungsstörung und reduzierter Eisenaufnahme [77]. Die rezente FINDCKD Studie zeigte, dass eine Eisenersatztherapie bei nichtdialysepflichtigen Patienten auch ohne Erythropoetingabe in über $2 / 3$ der Fälle eine adäquate Behandlung ohne Eskalationsnotwendigkeit darstellen kann [78]. Die Wirksamkeit der Erythropoetintherapie ist ebenfalls durch adäquate Eisenzufuhr gesteigert [79]. Die Ursache ist, dass Erythropoetin $\mathrm{zu}$ einer massiven Stimulation der Erythropoese führt, die den Eisenbedarf der Erythroblasten erheblich steigert. Im Rahmen der chronischen Entzündung kann aufgrund der Hepcidinwirkung ein ausreichender Eisenfluss von den Eisenspeichern zum Knochenmark nicht aufrechtgehalten werden; diesen Zustand bezeichnet man als funktionellen Eisenmangel. Der funktionelle Eisenmangel kann eine Thrombozytose hervorrufen [80, 81], und eine adäquate Eisenzufuhr kann die Plättchenanzahl wieder normalisieren [79]. Ein unbehandelter Eisenmangel erhöht die Mortalität bei Patienten mit chronischer Niereninsuffizienz [82, 83]. Es wird vermutet, dass die durch den funktionellen Eisenmangel hervorgerufene sekundäre Thrombozytose wesentlich zu der Erythropoetin-assoziierten Steigerung der Häufigkeit von kardiovaskulären Ereignissen beiträgt $[41,84]$. In einer großen retrospektiven Studie bei mit Erythropoetin und Eisen behandelten Hämodialysepatienten zeigten Streja et al., dass ein Hämoglobinwert >13 g/dl ohne begleitende Thrombozytose oder Eisenmangel nicht mit einer erhöhten Gesamtmortalität assoziiert war (OR 0,98); dagegen steigerten eine Transferrinsättigung $<20 \%$ und begleitende Thrombozytose die Mortalität (OR 2,29) [85]. Diese Ergebnisse legen nahe, dass die eisenmangelinduzierte Thrombozytose auch im Setting der renalen Anämie einen behebbaren Risikofaktor für Thromboseembolie-assoziierte Sterblichkeit darstellt.

\section{Thrombozytose und Thromboembolierisiko bei Malignompatienten - eine Rolle für Eisen?}

Die Assoziation zwischen malignen Erkrankungen und thromboembolische Komplikationen ist unumstritten. Mit einer Prävalenz bis $\mathrm{zu}>10 \%$ stellen Thromboembolien eine der wesentlichsten Ursachen für Morbidität und Mortalität in diesem Kollektiv dar. Wichtige Risikofaktoren sind die Art und Stadium der malignen Erkrankung, Chemotherapie, Hospitalisierung, Alter und Geschlecht [86]. Prospektive Studien zeigten, dass eine reaktive Thrombozytose bei Malignompatienten einen unabhängigen Risikofaktor für venöse Thromboembolien darstellt [49, 50]. In der großen prospektiven CATS Studie war die Thrombozytose mit einem HR für venöse Thromboembolien von 3,50 assoziiert [51]. Ferner wurde eine Thrombozytenaktivierung bei malignen Erkrankungen gezeigt, die ebenfalls mit einer Risikoerhöhung einhergeht [87].

Es ist nicht geklärt, inwiefern die reaktive Thrombozytose bei Malignomen eisenmangelbedingt sein kann. Eisenmangel und Eisenmangelanämie sind sehr häufig bei Patienten mit onkologischen Erkrankungen (>40 \% bzw. >30 \% Prävalenz) [88]. Eine Eisenersatztherapie ist die wichtigste therapeutische Maßnahme bei der krebsassoziierten Anämie; aufgrund der hepcidinbedingten Eisenverwertungsstörung ist allerdings das Ansprechen oft eingeschränkt, sodass eine begleitende Erythropoetintherapie, insbesondere bei der Chemotherapie-assoziierten Anämie, häufig notwendig wird [89, 90]. Ein erhöhtes Thromboserisiko (RR 1,67) unter Erythropoese-stimulierenden Agenten stellt auch bei Malignompatienten ein erhebliches Problem dar [91]. Wie bei der renalen Anämie verursacht Erythropoetin einen funktionellen Eisenmangel, sodass eine begleitende Eisentherapie benötigt wird, um ein optimales Ansprechen zu erzielen [92, 93]. Auch hier verursacht der funktionelle Eisenmangel eine Thrombozytose [94], die das venöse Thromboembolierisiko um das Vierfache erhöht [93]. Eine adäquate Eisenersatztherapie könnte somit auch bei Malignompatienten eine präventive Strategie darstellen, um Morbidität und Mortalität zu reduzieren.

Zusammenfassend finden sich vermehrt Hinweise, dass Eisenmangel eine reaktive Thrombozytose verursacht, die mehr als eine harmlose Laborabnormalität darstellt, sondern mit einem erhöhten Thromboembolierisiko in verschiedenen Populationen wie Patienten mit chronisch entzündlichen Darmerkrankungen, chronischer Niereninsuffizienz, malignen Erkrankungen, postoperativ oder bei Intensivpatienten verbunden ist. Wir spekulieren, dass die eisenmangelinduzierte Thrombozytose evolutionär entstanden ist, um einen Blutverlust, der ja einen Eisenmangel verursacht, zu minimieren. Unter den oben genannten Bedingungen kann dies aber zu einer potentiell gefährlichen prothrombogenen Risikokonstellation führen. Es liegt nahe, dass eine Eisenersatztherapie eine wichtige Maßnahme zur Thromboembolierisikoreduktion darstellen könnte. Prospektive Studien sind notwendig, um diese Hypothese zu überprüfen.

Open access funding provided by Medical University of Vienna.

Interessenkonflikt R. Evstatiev gibt an, dass kein Interessenkonflikt besteht.

Open Access Dieser Artikel wird unter der Creative Commons Namensnennung 4.0 International Lizenz (http:// creativecommons.org/licenses/by/4.0/deed.de) veröffent- 
licht, welche die Nutzung, Vervielfältigung, Bearbeitung, Verbreitung und Wiedergabe in jeglichem Medium und Format erlaubt, sofern Sie den/die ursprünglichen Autor(en) und die Quelle ordnungsgemäß nennen, einen Link zur Creative Commons Lizenz beifügen und angeben, ob Änderungen vorgenommen wurden.

\section{Literatur}

1. WHO, UNICEF, UNU. Iron deficiency anemia: assessment, prevention and control 2001. http://whqlibdoc.who.int/ hq/2001/WHO_NHD_01.3.pdf.Zugriffen: 12.09.2016

2. Evstatiev R, Gasche C. Iron sensing and signalling. Gut. 2012;61(6):933-52.

3. Yadav D, Chandra J. Iron deficiency: beyond anemia. Indian JPediatr. 2011;78(1):65-72.

4. Opal SM. Phylogenetic and functional relationships between coagulation and the innate immune response. Crit Care Med. 2000;28(9Suppl):77-80.

5. Weyrich AS, Lindemann S, Zimmerman GA. The evolving role of platelets in inflammation. J Thromb Haemostasis. 2003;1(9):1897-905.

6. Geddis AE, Fox N, Tkacenko E, Kaushansky K. Endomitotic megakaryocytesthatformabipolarspindle exhibitcleavage furrow ingression followed by furrow regression. Cell Cycle. 2007;6(4):455-60.

7. Lordier L, Jalil A, Aurade F, Larbret F, Larghero J, Debili $\mathrm{N}$, et al. Megakaryocyte endomitosis is a failure of late cytokinesis related to defects in the contractile ring and Rho/Rocksignaling. Blood.2008;112(8):3164-74.

8. Lordier L, Pan J, Naim V, Jalil A, Badirou I, Rameau P, et al. Presence of a defect in karyokinesis during megakaryocyte endomitosis. Cell Cycle. 2012;11(23):4385-9.

9. RavidK, LuJ,ZimmetJM, Jones MR. Roads to polyploidy: the megakaryocyte example. J Cell Physiol. 2002;190(1):7-20.

10. EvstatievR, BukatyA, JimenezK, Kulnigg-DabschS, Surman L, Schmid W, et al. Iron deficiency alters megakaryopoiesis and platelet phenotype independent of thrombopoietin. AmJ Hematol.2014;89(5):524-9.

11. Buss DH, Cashell AW, O'Connor ML, Richards F 2nd, Case LD. Occurrence, etiology, and clinical significance of extreme thrombocytosis: a study of 280 cases. Am J Med. 1994;96(3):247-53.

12. Griesshammer M, Bangerter M, Sauer T, Wennauer R, Bergmann L, Heimpel H. Aetiology and clinical significance of thrombocytosis: analysis of 732 patients with an elevated platelet count. J Intern Med. 1999;245(3):295-300.

13. Schafer AI. Thrombocytosis. N Engl J Med. 2004;350(12): 1211-9.

14. Tefferi A, Ho TC, Ahmann GJ, Katzmann JA, Greipp PR. Plasma interleukin-6 and C-reactive protein levels in reactive versus clonal thrombocytosis. Am J Med. 1994;97(4):374-8.

15. Dan K. Thrombocytosis in iron deficiency anemia. Intern Med.2005;44(10):1025-6.

16. Gross S, Keefer V, Newman AJ. The platelets in irondeficiency anemia. I. The responce to oral and parenteral iron. Pediatrics. 1964;34:315-23.

17. Kasper CK, Whissell DY, Wallerstein RO. Clinical aspects of iron deficiency. JAMA. 1965;191:359-63.

18. Keung YK, Owen J. Iron deficiency and thrombosis: literature review. Clin Appl Thromb Hemost. 2004;10(4):387-91.

19. Sandoval C. Thrombocytosis in children with iron deficiency anemia: series of 42 children. J Pediatr Hematol Oncol. 2002;24(7):593.
20. Schloesser LL, Kipp MA, Wenzel FJ. Thrombocytosis in iron deficiency anemia. J Lab Clin Med. 1965;66:107-14.

21. Kulnigg-Dabsch S, Evstatiev R, Dejaco C, Gasche C. Effect of iron therapy on platelet counts in patients with inflammatory bowel disease-associated anemia. PLOS ONE. 2012;7(4):e34520.

22. Kulnigg-Dabsch S, Schmid W, Howaldt S, Stein J, Mickisch $\mathrm{O}$, Waldhor $\mathrm{T}$, et al. Iron deficiency generates secondary thrombocytosis and platelet activation in IBD: the randomized, controlled thromboVIT trial. Inflamm Bowel Dis. 2013;19(8):1609-16, Jul.

23. Kadikoylu G, Yavasoglu I, Bolaman Z, Senturk T. Platelet parameters in women with iron deficiency anemia. J Natl Med Assoc. 2006;98(3):398-402.

24. ChoiSI, SimoneJV.Plateletproduction in experimental iron deficiency anemia. Blood. 1973;42(2):219-28.

25. Choi SI, Simone JV, Jackson CW. Megakaryocytopoiesis in experimental iron deficiency anemia. Blood. 1974;43(1):111-20.

26. Garg SK, Weiner M. Thrombocyte and megathrombocyte kinetics during thrombocytosis induced by acute and chronic blood loss and by iron-deficiency diet. Haemostasis. 1972;1(3-4):121.

27. Holter PH, Refsum HE. Postnatal anemia and thrombocytosis in suckling rabbits: influence of delayed weaning and iron supplies. Pediatr Hematol Oncol. 1988;5(3):157-68.

28. Karpatkin S, Garg SK, Freedman ML. Role of iron as a regulator of thrombopoiesis. Am J Med. 1974;57(4):521-5.

29. Kuku I, Kaya E, Yologlu S, Gokdeniz R, Baydin A. Platelet counts in adults with iron deficiency anemia. Platelets. 2009;20(6):401-5.

30. Perlman MK, Schwab JG, Nachman JB, Rubin CM. Thrombocytopenia in children with severe iron deficiency. J Pediatr Hematol Oncol. 2002;24(5):380-4.

31. Park MJ, Park PW, Seo YH, Kim KH, Park SH, Jeong JH, etal. The relationshipbetween iron parameters and platelet parameters in women with iron deficiency anemia and thrombocytosis. Platelets. 2013;24(5):348-51.

32. Akan H, Guven N, Aydogdu I, Arat M, Beksac M, Dalva K. Thrombopoietic cytokines in patients with iron deficiency anemia with or without thrombocytosis. Acta Haematol. 2000;103(3):152-6.

33. Ceresa IF, Noris P, Ambaglio C, Pecci A, Balduini CL. Thrombopoietin is not uniquely responsible for thrombocytosis in inflammatory disorders. Platelets. 2007;18(8):579-82.

34. Papa A, Danese S, Piccirillo N, Toriani-Terenzi C, Bartolozzi F, Piscaglia AC, et al. Thrombopoietin serum levels in patients with inflammatory bowel disease with and without previous thromboembolic events. Hepatogastroenterology. 2003;50(49):132-5.

35. Beguin Y, Loo M, R'ZikS, Sautois B, LejeuneF, Rorive G, et al. Effect of recombinant human erythropoietin on platelets in patients with anemia of renal failure: correlation of platelet count with erythropoietic activity and iron parameters. Eur JHaematol. 1994;53(5):265-70.

36. Broudy VC, Lin NL, Kaushansky K. Thrombopoietin (c-mpl ligand) acts synergistically with erythropoietin, stem cell factor, and interleukin-11 to enhance murine megakaryocyte colony growth and increases megakaryocyte ploidy in vitro. Blood. 1995;85(7):1719-26.

37. Racke FK. EPO and TPO sequences do not explain thrombocytosis in iron deficiency anemia. J Pediatr HematolOncol. 2003;25(11):919.

38. Klimchenko O, Mori M, Distefano A, Langlois T, Larbret F, Lecluse $\mathrm{Y}$, et al. A common bipotent progenitor generates the erythroid and megakaryocyte lineages in embryo- 
nic stem cell-derived primitive hematopoiesis. Blood. 2009;114(8):1506-17.

39. Bilic E, Bilic E. Amino acid sequence homology of thrombopoietin and erythropoietin may explain thrombocytosis in children with iron deficiency anemia. J Pediatr Hematol Oncol. 2003;25(8):675-6.

40. Geddis AE, Kaushansky K. Cross-reactivity between erythropoietin and thrombopoietin at the level of Mpl does not account for the thrombocytosis seen in iron deficiency. J Pediatr Hematol Oncol. 2003;25(11):919-20.

41. Dahl NV, Henry DH, Coyne DW. Thrombosis with erythropoietic stimulating agents-does iron-deficient erythropoiesis play a role? Semin Dial. 2008;21(3):210-1.

42. JimenezK, KhareV,EvstatievR, Kulnigg-DabschS, Jambrich $\mathrm{M}, \mathrm{Strobl} \mathrm{H}$, et al. Increased expression of HIF2alpha during iron deficiency-associated megakaryocytic differentiation. JThromb Haemostasis. 2015;13(6):1113-27.

43. Robinson KA, O’Donnell ME, Pearson D, Kriegshauser JS, Odeleye M, Kalkbrenner K, et al. Portomesenteric venous thrombosis following major colon and rectal surgery: incidence and risk factors. Surg Endosc. 2015;29(5):1071-9.

44. Schmuziger M, Christenson JT, Maurice J, Simonet F, Velebit V. Reactive thrombocytosis after coronary bypass surgery. An important risk factor. Eur J Cardiothorac Surg. 1995;9(7):393-7, discussion 7-8.

45. Kashuk JL, Moore EE, Johnson JL, Biffl WL, Burlew CC, Barnett C, et al. Progressive postinjury thrombocytosis is associated with thromboembolic complications. Surgery. 2010;148(4):667-74, discussion 74-5.

46. Ho KM, Chavan S. Prevalence of thrombocytosis in critically ill patients and its association with symptomatic acute pulmonary embolism. A multicentre registry study. Thromb Haemost. 2013;109(2):272-9.

47. Ho KM, Yip CB, Duff O. Reactive thrombocytosis and risk of subsequent venous thromboembolism: a cohort study. J Thromb Haemostasis. 2012;10(9):1768-74.

48. Pate A, Baltazar GA, Labana S, Bhagat T, Kim J, Chendrasekhar A. Systemic inflammatory response syndrome and platelet count $\rangle /=250 \times 10(9)$ are associated with venous thromboembolic disease. IntJ Gen Med. 2015;8:37-40.

49. Khorana AA, Francis CW, Culakova E, Lyman GH. Risk factors for chemotherapy-associated venous thromboembolism in a prospective observational study. Cancer. 2005;104(12):2822-9.

50. Khorana AA, Kuderer NM, Culakova E, Lyman GH, Francis CW. Development and validation of a predictive model for chemotherapy-associated thrombosis. Blood. 2008;111(10):4902-7.

51. Simanek R, Vormittag R, Ay C, Alguel G, Dunkler D, Schwarzinger I, et al. High platelet count associated with venous thromboembolism in cancer patients: results from the Vienna Cancer and Thrombosis Study (CATS). JThromb Haemost. 2010;8(1):114-20.

52. Cooke NM, Egan K, McFadden S, Grogan L, Breathnach OS, O'Leary J, et al. Increased platelet reactivity in patients with late-stage metastatic cancer. Cancer Med. 2013;2(4):564-70.

53. DuffOC, Ho KM, MayburySM. In vitrothrombotic tendency of reactive thrombocytosis in critically ill patients: a prospective case-control study. Anaesth Intensive Care. 2012;40(3):472-8.

54. Harr JN, Moore EE, Chin TL, Ghasabyan A, Gonzalez E, Wohlauer MV, et al. Platelets are dominant contributors to hypercoagulability after injury. J Trauma Acute Care Surg. 2013;74(3):756-62, discussion 62-5.

55. Azab SF, Abdelsalam SM, Saleh SH, Elbehedy RM, Lotfy SM, Esh AM, et al. Iron deficiency anemia as a risk factor for cerebrovascular events in early childhood: a case-control study. Ann Hematol. 2014;93(4):571-6.

56. Chang YL, Hung SH, Ling W, Lin HC, Li HC, Chung SD. Association between ischemic stroke and iron-deficiency anemia: a population-based study. PLOS ONE. 2013;8(12):e82952.

57. Dubyk MD, Card RT, Whiting SJ, Boyle CA, Zlotkin SH, Paterson PG. Iron deficiency anemia prevalence at first stroke or transient ischemic attack. Can J Neurol Sci. 2012;39(2):189-95.

58. Gillum RF, Sempos CT, Makuc DM, Looker AC, Chien CY, Ingram DD. Serum transferrin saturation, stroke incidence, and mortality in women and men. The NHANES I epidemiologic followup study. National Health and Nutrition Examination Survey. Am JEpidemiol. 1996;144(1):59-68.

59. Katsanos AH, Kosmidou M, Giannopoulos S, Katsanos $\mathrm{KH}$, Tsivgoulis G, Kyritsis AP, et al. Cerebral arterial infarction in inflammatory bowel diseases. Eur J Intern Med.2014;25(1):37-44.

60. Hung SH, Lin HC, Chung SD. Association between venous thromboembolism and iron-deficiency anemia: a population-based study. Blood Coagul Fibrinolysis. 2015;26(4):368-72.

61. Potaczek DP, Jankowska EA, Wypasek E, Undas A. Iron deficiency: a novel risk factor of recurrence in patients after unprovoked venous thromboembolism. Pol Arch Med Wewn.2016;126 doi:10.20452/pamw.3311.

62. Stolz E, Valdueza JM, Grebe M, Schlachetzki F, Schmitt $\mathrm{E}$, Madlener K, et al. Anemia as a risk factor for cerebral venous thrombosis? An old hypothesis revisited. Results of a prospective study. J Neurol.2007;254(6):729-34.

63. Grainge MJ, West J, Card TR. Venous thromboembolism during active disease and remission in inflammatory bowel disease: a cohort study. Lancet. 2010;375(9715):657-63.

64. Miehsler W, Reinisch W, Valic E, Osterode W, Tillinger W, Feichtenschlager T, et al. Is inflammatory bowel disease an independent and disease specific risk factor for thromboembolism? Gut. 2004;53(4):542-8.

65. Novacek G, Weltermann A, Sobala A, Tilg H, Petritsch W, Reinisch W, et al. Inflammatory bowel disease is a risk factor for recurrent venous thromboembolism. Gastroenterology. 2010;139(3):779-87,787 e1.

66. Filmann N, Rey J, Schneeweiss S, Ardizzone S, Bager P, Bergamaschi G, et al. Prevalence of anemia in inflammatory bowel diseases in european countries: a systematic review and individual patient data meta-analysis. Inflamm Bowel Dis. 2014;20(5):936-45.

67. Kulnigg S, Gasche C. Systematic review: managing anaemia in Crohn's disease. Aliment Pharmacol Ther. 2006;24(11-12):1507-23.

68. Harries AD, Fitzsimons E, Fifield R, Dew MJ, Rhoades J. Platelet count: a simple measure of activity in Crohn's disease. Br Med J. 1983;286(6376):1476.

69. Talstad I, Rootwelt K, Gjone E. Thrombocytosis in ulcerative colitis and Crohn's disease. Scand J Gastroenterol. 1973;8(2):135-8.

70. Heits F, Stahl M, Ludwig D, Stange EF, Jelkmann W. Elevated serum thrombopoietin and interleukin- 6 concentrations in thrombocytosis associated with inflammatory bowel disease. J Interferon Cytokine Res. 1999;19(7):757-60.

71. Voudoukis E, Karmiris K, Oustamanolakis P, Theodoropoulou A, Sfiridaki A, Paspatis GA, et al. Association between thrombocytosis and iron deficiency anemia in inflammatory bowel disease. Eur J Gastroenterol Hepatol. 2013; doi:10. 1097/meg.0b013e328363e354. 
72. Webberley MJ, Hart MT, Melikian V. Thromboembolism in inflammatory bowel disease: role of platelets. Gut. 1993;34(2):247-51.

73. Besarab A, Bolton WK, Browne JK, Egrie JC, Nissenson AR, Okamoto DM, et al. The effects of normal as compared with low hematocrit values in patients with cardiac disease who are receiving hemodialysis and epoetin. N Engl J Med. 1998;339(9):584-90.

74. Drueke TB, Locatelli F, Clyne N, Eckardt KU, Macdougall IC, Tsakiris D, et al. Normalization of hemoglobin level in patients with chronic kidney disease and anemia. N Engl J Med. 2006;355(20):2071-84.

75. Phrommintikul A, Haas SJ, Elsik M, Krum H. Mortality and target haemoglobin concentrations in anaemic patients with chronic kidney disease treated with erythropoietin: a meta-analysis. Lancet. 2007;369(9559):381-8.

76. Singh AK, Szczech L, Tang KL, Barnhart H, Sapp S, Wolfson $\mathrm{M}$, et al. Correction of anemia with epoetin alfa in chronic kidney disease. N Engl J Med. 2006;355(20):2085-98.

77. Macdougall IC, Bircher AJ, Eckardt KU, Obrador GT, Pollock $\mathrm{CA}$, Stenvinkel P, et al. Iron management in chronic kidney disease: conclusions from a "Kidney Disease: Improving Global Outcomes" (KDIGO) Controversies Conference. Kidney Int. 2016;89(1):28-39.

78. Macdougall IC, Bock AH, Carrera F, Eckardt KU, Gaillard C, van Wyck D, et al. FIND-CKD: a randomized trial of intravenous ferric carboxymaltose versus oral iron in patients with chronic kidney disease and iron deficiency anaemia. NephrolDial Transplant. 2014;29(11):2075-84.

79. Coyne DW, Kapoian T, Suki W, Singh AK, Moran JE, Dahl $\mathrm{NV}$, et al. Ferric gluconate is highly efficacious in anemic hemodialysis patients with high serum ferritin and low transferrin saturation: results of the Dialysis Patients' Response to IV Iron with Elevated Ferritin (DRIVE) Study. JAm Soc Nephrol.2007;18(3):975-84.

80. Beguin Y. Erythropoietin and platelet production. Haematologica. 1999;84(6):541-7.

81. Loo M, Beguin Y. The effect of recombinant human erythropoietin on platelet counts is strongly modulated by the adequacy of iron supply. Blood. 1999;93(10):3286-93.

82. Kalantar-Zadeh K, McAllister CJ, Lehn RS, Liu E, Kopple JD. A low serum iron level is a predictor of poor outcome in hemodialysis patients. Am J Kidney Dis. 2004;43(4):671-84.

83. Kalantar-Zadeh K, Regidor DL, McAllister CJ, Michael B, Warnock DG. Time-dependent associations between iron and mortality in hemodialysis patients. J Am Soc Nephrol. 2005;16(10):3070-80.

84. Besarab A, Horl WH, Silverberg D. Iron metabolism, iron deficiency, thrombocytosis, and the cardiorenal anemia syndrome. Oncologist. 2009;14(Suppl 1):22-33.

85. Streja E, Kovesdy CP, Greenland S, Kopple JD, McAllister CJ, Nissenson AR, et al. Erythropoietin, iron depletion, and relative thrombocytosis: a possible explanation for hemoglobin-survival paradoxin hemodialysis. Am J Kidney Dis. 2008;52(4):727-36.

86. Konigsbrugge O, Pabinger I, Ay C. Risk factors for venous thromboembolism in cancer: novel findings from the Vienna Cancer and Thrombosis Study (CATS). Thromb Res. 2014;133(Suppl2):39-43.

87. Ay C, Dunkler D, Marosi C, ChiriacAL, Vormittag R, Simanek $\mathrm{R}$, et al. Prediction of venous thromboembolism in cancer patients. Blood.2010;116(24):5377-82.

88. Ludwig H, Muldur E, Endler G, Hubl W. Prevalence of iron deficiency across different tumors and its association with poor performance status, disease status and anemia. Ann Oncol.2013;24(7):1886-92.
89. RizzoJD, Brouwers M, HurleyP, Seidenfeld J, Somerfield MR, Temin S. American society of clinical oncology/american society of hematology clinical practice guideline update on the use of epoetin and darbepoetin in adult patients with cancer.J OncolPract. 2010;6(6):317-20.

90. Schrijvers D, De Samblanx H, Roila F, Group EGW. Erythropoiesis-stimulating agents in the treatment of anaemia in cancer patients: ESMO Clinical Practice Guidelines for use. Ann Oncol.2010;21(Suppl5):v244-v247.

91. Bohlius J, Wilson J, Seidenfeld J, Piper M, Schwarzer G, Sandercock J, et al. Recombinant human erythropoietins and cancer patients: updated meta-analysis of 57 studies including 9353 patients. J Natl Cancer Inst. 2006;98(10):708-14.

92. Auerbach M, Ballard H, Trout JR, McIlwain M, Ackerman A, Bahrain $\mathrm{H}$, et al. Intravenous iron optimizes the response to recombinanthuman erythropoietin in cancer patients with chemotherapy-related anemia: a multicenter, open-label, randomized trial. J Clin Oncol. 2004;22(7):1301-7.

93. Henry DH, Dahl NV, Auerbach M, Tchekmedyian S, Laufman LR. Intravenous ferric gluconate significantly improves response to epoetin alfa versus oral iron or no iron in anemic patients with cancer receiving chemotherapy. Oncologist. 2007;12(2):231-42.

94. Henry DH, Dahl NV, Auerbach MA. Thrombocytosis and venous thromboembolism in cancer patients with chemotherapy induced anemia may be related to ESA induced iron restricted erythropoiesis and reversed by administration of IV iron. Am J Hematol. 2012;87(3):308-10.

95. Knizley H Jr., Noyes WD. Iron deficiency anemia, papilledema, thrombocytosis, and transient hemiparesis. Arch Intern Med. 1972;129(3):483-6.

96. Alexander MB. Iron deficiency anemia, thrombocytosis, and cerebrovascular accident. South Med J. 1983;76(5):662-3.

97. Heller DS, Pervez NK, Kleinerman J. Fatal cerebrovascular thrombosis in a young woman: an unusual complication associated with hypochromic anemia and thrombocytosis following surgery. Mt Sinai J Med. 1988;55(4):318-20.

98. Ready WK, Lowry NJ. Anemia causing cerebral infarction in a child. Can Med Assoc J. 1989;140(3):303-4.

99. Aoki N, Sakai T. Cerebral sinus thrombosis in patients with severe iron deficiency anaemia due to myoma uteri. Acta Neurochir(Wien). 1989;97(3-4):131-4.

100. Bruggers CS, Ware R, Altman AJ, RourkMH, Vedanarayanan $\mathrm{V}$, Chaffee S. Reversible focal neurologic deficits in severe iron deficiency anemia. J Pediatr. 1990;117(3):430-2.

101. Saxena VK, Brands C, Crols R, Moens E, Marien P, de Deyn PP. Multiple cerebral infarctions in a young patient with secondary thrombocythemia due to iron deficiency anemia. Acta Neurol (Napoli). 1993;15(4):297-302.

102. Akins PT, Glenn S, Nemeth PM, Derdeyn CP. Carotid artery thrombus associated with severe iron-deficiency anemia and thrombocytosis. Stroke. 1996;27(5):1002-5.

103. Matsuoka Y, Hayasaka S, Yamada K. Incomplete occlusion of central retinal artery in a girl with iron deficiency anemia. Ophthalmol J Int. 1996;210(6):358-60.

104. Hartfield DS, Lowry NJ, Keene DL, Yager JY. Iron deficiency: a cause of stroke in infants and children. Pediatr Neurol. 1997;16(1):50-3.

105. Swann IL, Kendra JR. Severe iron deficiency anaemia and stroke. Clin Lab Haematol. 2000;22(4):221-3.

106. Kacer B, Hattenbach LO, Horle S, Scharrer I, Kroll P, Koch F. Central retinal vein occlusion and nonarteritic ischemic optic neuropathy in 2 patients with mild iron deficiency anemia. Ophthalmol J Int. 2001;215(2):128-31.

107. Standridge S, de los Reyes E. Inflammatory bowel disease and cerebrovascular arterial and venous thromboembolic 
events in 4 pediatric patients: a case series and review of the literature. JChild Neurol. 2008;23(1):59-66.

108. Williams B, Morton C. Cerebral vascular accident in a patient with reactive thrombocytosis: a rare cause of stroke. Am J Med Sci. 2008;336(3):279-81.

109. Wiesen JA, Kurtz LE, Katz S. Widespread occlusive vascular disease in a Crohn's disease patient with profound thrombocytosis. JClin Gastroenterol.2006;40(9):863-4.

110. Bukharovich IF, Wever-Pinzon O, Shah A, Todd G, Chaudhry FA, Sherrid MV. Arterial embolism caused by large mobile aortic thrombus in the absence of atherosclerosis, associated with iron deficiency anemia. Echocardiography. 2012;29(3):369-72.

111. Batur Caglayan HZ, Nazliel B, Irkec C, Dumlu A, Filiz A, Panpalli Ates M. Iron-deficiency anemia leading to transient ischemic attacks due to Intraluminal carotid artery thrombus. Case Rep Neurol Med. 2013;2013:813415. doi:10.1155/2013/813415.

112. Naito H, Naka H, Kanaya Y, Yamazaki Y, Tokinobu H. Two cases of acute ischemic stroke associated with iron deficiency anemia due to bleeding from uterine fibroids in middle-aged women. Intern Med. 2014;53(21):2533-7.

113. Kirkham TH, Wrigley PF, Holt JM. Central retinal vein occlusion complicating iron deficiency anaemia. $\mathrm{Br} \mathrm{J}$ Ophthalmol. 1971;55(11):777-80.

114. Belman AL, Roque CT, Ancona R, Anand AK, Davis RP. Cerebral venous thrombosis in a child with iron deficiency anemia and thrombocytosis. Stroke. 1990;21(3):488-93.

115. Shibuya Y, Hayasaka S. Central retinal vein occlusion in a patient with anorexia nervosa. Am J Ophthalmol. 1995;119(1):109-10.

116. Meena AK, Naidu KS, Murthy JM. Cortical sinovenous thrombosis in a child with nephrotic syndrome and iron deficiency anaemia. Neurol India. 2000;48(3):292-4.

117. Benedict SL, Bonkowsky JL, Thompson JA, van Orman CB, Boyer RS, Bale JF Jr., et al. Cerebral sinovenous thrombosis in children: another reason to treat iron deficiency anemia. JChild Neurol.2004;19(7):526-31.

118. Kinoshita Y, Taniura S, Shishido H, Nojima T, Kamitani H, Watanebe T. Cerebral venous sinus thrombosis associated with iron deficiency: two case reports. Neurol Med Chir (Tokyo).2006;46(12):589-93.

119. Balci K, Utku U, Asil T, Buyukkoyuncu N. Deep cerebral vein thrombosis associated with iron deficiency anaemia in adults. JClin Neurosci. 2007;14(2):181-4.
120. Ogata T, Kamouchi M, Kitazono T, Kuroda J, Ooboshi H, Shono T, et al. Cerebral venous thrombosis associated with iron deficiency anemia. J Stroke Cerebrovasc Dis. 2008;17(6):426-8.

121. Habis A, Hobson WL, Greenberg R. Cerebral sinovenous thrombosis in a toddler with iron deficiencyanemia. Pediatr Emerg Care. 2010;26(11):848-51.

122. Nicastro N, Schnider A, Leemann B. Iron-deficiency anemia as a rare cause of cerebral venous thrombosis and pulmonary embolism. Case Rep Med. 2012;2012:497814. doi:10.1155/2012/497814

123. Beri S, Khan A, Hussain N, Gosalakkal J. Severe anemia causing cerebral venous sinus thrombosis in an infant. JPediatr Neurosci. 2012;7(1):30-2.

124. Zhu XQ, Cao L. Cerebral venous sinus thrombosis following diagnostic curettage in a patient with uterine fibroid. Case Rep Neurol Med. 2014;2014:270654. doi:10.1155/2014/ 270654.

125. Nishioka K, Tanaka R, Tsutsumi S, Yamashiro K, Nakahara $\mathrm{M}$, Shimura $\mathrm{H}$, et al. Cerebral dural sinus thrombosis associated with adenomyosis: a case report. J Stroke Cerebrovasc Dis. 2014;23(7):1985-7.

126. Corrales-Medina FF, Grant L, Egas-Bejar D, Valdivia-Ascuna Z, Rodriguez N, Mancias P. Cerebral sinovenous thrombosis associated with iron deficiency anemia secondary to severe menorrhagia: a case report. J Child Neurol. 2014;29(9):NP62-NP64.

127. Choe Y, Lee JB, Kim YJ, Choi IS. Cerebral venous sinus thrombosis and venous hemorrhagic infarction in a young woman. Ann Rehabil Med.2014;38(5):698-701.

128. Finkel L, Piantino J, Goldstein J, Wainwright MS. Venous stroke and status epilepticus due to milk-induced anemia in a child. Pediatr Emerg Care. 2015;31 (2):129-31, Feb.

129. Perlman MK, Schwab JG, Nachman JB, Rubin CM. Thrombocytopenia in children with severe iron deficiency. J Pediatr HematolOncol.20026/2002;24(5):380-4.

130. Sladic-Simic D, Martinovitch PN, Zivkovic N, Pavic D, Martinovic J, Kahn M, et al. A thalassemia-like disorder in Belgrade laboratory rats. Annals of the New York Academy of Sciences. 1969 Nov 20;165(1):93-9.

131. Du X, She E, Gelbart T, Truksa J, Lee P, Xia Y, et al. The serine protease TMPRSS6 is required to sense iron deficiency. Science. 2008 May23;320(5879):1088-92. 